Mon. Not. R. Astron. Soc. 000, 1 12 (2016) Printed 9 October $2018 \quad$ (MN [aT $\mathrm{E}_{\mathrm{E}} \mathrm{X}$ style file v2.2)

\title{
Staying away from the bar: the local dynamical signature of slow and fast bars in the Milky Way
}

\author{
Giacomo Monari ${ }^{1} *$, Benoit Famaey ${ }^{1}$, Arnaud Siebert ${ }^{1}$, Aurore Duchateau ${ }^{1,2}$, \\ Thibault Lorscheider ${ }^{1,2}$ and Olivier Bienaymé ${ }^{1}$ \\ 1 Observatoire astronomique de Strasbourg, Université de Strasbourg, CNRS UMR 7550, 11 rue de l'Université, 67000 Strasbourg, France \\ ${ }^{2}$ UFR de Mathématique et d'Informatique, Université de Strasbourg, 7 rue René Descartes, 67084 Strasbourg, France
}

Released 2016

\begin{abstract}
Both the three-dimensional density of red clump giants and the gas kinematics in the inner Galaxy indicate that the pattern speed of the Galactic bar could be much lower than previously estimated. Here, we show that such slow bar models are unable to reproduce the bimodality observed in local stellar velocity space. We do so by computing the response of stars in the Solar neighbourhood to the gravitational potential of slow and fast bars, in terms of their perturbed distribution function in action-angle space up to second order, as well as by identifying resonantly trapped orbits. We also check that the bimodality is unlikely to be produced through perturbations from spiral arms, and conclude that, contrary to gas kinematics, local stellar kinematics still favour a fast bar in the Milky Way, with a pattern speed of the order of almost twice (and no less than 1.8 times) the circular frequency at the Sun's position. This leaves open the question of the nature of the long flat extension of the bar in the Milky Way.
\end{abstract}

Key words: Galaxy: kinematics and dynamics - Galaxy: disc - Galaxy: solar neighborhood - Galaxy: structure - Galaxy: evolution

\section{INTRODUCTION}

The Milky Way is a barred galaxy. This conclusion can be readily established from the gas kinematics in the inner Galaxy (e.g., de Vaucouleurs 1964 Binney et al. 1991), as well as from near-infrared photometry (Binney et al. 1997). Nevertheless, and rather surprisingly, the structural parameters of the Milky Way bar, in particular its strength, orientation and pattern speed, are still very poorly constrained.

Almost two decades ago, based on photometry and gas kinematics arguments, a consensus emerged for a fast bar with corotation $(\mathrm{CR})$ around $\sim 3.5 \mathrm{kpc}$ (e.g., Binney et al. 1997, Bissantz et al. 2003), i.e. a perturbation pattern speed $\Omega_{\mathrm{b}} \approx 1.9 \Omega_{0}$ where $\Omega_{0}$ is the local rotational frequency at the Sun's radius $R_{0}$, and an angle between the bar major axis and the Galactic centre-Sun direction of $\phi_{\mathrm{b}} \sim 25^{\circ}$. This pattern speed would place the Sun just outside the outer Lindblad resonance (OLR) of the bar, and the kinematical signature associated with this position indeed appears to be present in the stellar phase-space distribution in the Solar neighbourhood (e.g., Dehnen 1999a, 2000 Famaey et al. 2005, Minchev et al. 2007| Bovy |2010, Quillen et al.|2011.

* Email: giacomo.monari@astro.unistra.fr see also Section 2, as well as possibly in large-scale stellar velocity fluctuations (Monari et al. 2014; Bovy et al. 2015).

However, from the photometric point of view, the situation has recently changed quite dramatically, since Wegg \& Gerhard (2013) and Wegg et al. (2015) measured the threedimensional density of red clump giants in the inner Galaxy by combining various recent photometric surveys. They concluded that the Milky Way contains a central box/peanut bulge (Combes et al. 1990, Athanassoula 2005) which is the vertical extension of a longer, flatter bar, oriented at an angle of $\phi_{\mathrm{b}} \sim 27^{\circ}$ from the Galactic centre-Sun direction, but reaching out to a radius $R_{\mathrm{b}} \sim 5 \mathrm{kpc}$. Since the bar cannot physically extend beyond its corotation, this limits the pattern speed of the bar. Simulated bars are usually rather shorter than their corotation, and indeed by constructing dynamical models reproducing this new bar density as well as the stellar kinematics from the BRAVA survey, the pattern speed was estimated to be of the order of $\Omega_{\mathrm{b}} \approx \Omega_{0}$ (Portail et al. 2015) placing the bar corotation very near to the Sun. Two independent subsequent re-analyses of gas kinematics in the inner Galaxy by Sormani et al. (2015) and Li et al. (2016) then favored slightly higher pattern speeds, of the order of $\Omega_{\mathrm{b}} \approx 1.45 \Omega_{0}$ and $\Omega_{\mathrm{b}} \approx 1.2 \Omega_{0}$ respectively, but both still much lower than the older estimate $\Omega_{\mathrm{b}} \approx 1.9 \Omega_{0}$.

Given this state of affairs, we now study in the present contribution the effect of such low pattern speeds on stellar 
kinematics in the Solar neighbourhood, which was previously considered a strong argument in favor of a fast bar. In Section 2, we briefly review the characteristic observational signatures of non-axisymmetries in the Solar neighbourhood, and more specifically the prominent Hercules moving group in velocity space. We then review in Section 3 the expected theoretical form (Monari et al. 2016a, hereafter M16) of the first order response of the phase-space distribution function (DF) in the presence of non-axisymmetric potential perturbations. We then extend this analysis up to second order, and also identify the location of resonantly trapped orbits in local velocity space. We subsequently confront the predictions to observations in the case of bars with low (Section 4 and high (Section 5 pattern speed. Conclusions are drawn in Section 6

\section{THE BIMODAL LOCAL VELOCITY SPACE}

The study of the fine structure of stellar velocity space in the Solar Neighbourhood dates back to more than a century and the work of Proctor (1869) and Kapteyn (1905), leading to the discovery of the Hyades and Ursa Major moving groups. These are spatially unbound groups of stars sharing similar velocities, but not the same age nor chemical composition (e.g., Famaey et al. 2005, 2007, 2008), and hence most likely associated with perturbations by disc non-axisymmetries. Another prominent structure in velocity space for late-type stars was discovered by Eggen (1958) and Blaauw (1970): it is located further away from the centre of the $U V$-space ${ }^{1}$ than the Hyades and Ursa Major groups, with velocities similar to the $\xi$ Herculis star. This structure creates a true secondary mode in local velocity space. This bimodal velocity space is thus separated into a main (high- $V$ ) mode, and a secondary (low- $V$ ) mode made up of this large moving group, referred to as the Hercules stream, or Hercules moving group. Analysis of the chemical abundances of this moving group revealed properties of a mixed population of thin and thick disc stars, with a prevalence of metal-rich thin disc stars, consistent with a dynamical perturbation from a non-axisymmetry of the potential (e.g., Soubiran \& Girard 2005, Bensby et al. 2007, Ramya et al.|2016. Antoja et al. 2016 in preparation). With a wavelet analysis of the $U V$-plane based on Hipparcos astrometric data combined with CORAVEL radial velocities for $\mathrm{K}$ and $\mathrm{M}$ giants (Famaey et al. 2005), the structure has actually been identified by Famaey et al. (2008) as a double-peak structure centred on $(U, V) \simeq(-35,-51) \mathrm{km} \mathrm{s}^{-1}$ for the first ' $\mathrm{H} 1$ ' peak, and $(U, V) \simeq(-57,-51) \mathrm{km} \mathrm{s}^{-1}$ for the second ' $\mathrm{H} 2$ ' peak.

The location of the Hercules moving group in velocity space has been shown by Dehnen (1999b, 2000) and Mühlbauer \& Dehnen (2003) to be a natural dynamical signature of the bar if the OLR radius is located just inside the Solar position, since the orbits aligned with the orientation of the bar at the Sun (the main mode) co-exist with orbits anti-aligned with the bar (Athanassoula et al. 1983), which

1 Here we use the common notation of Galactic Astronomy in which, at the position of the Sun in the Galactic plane, $U$ is the stellar velocity towards the Galactic centre, and $V$ the velocity in the direction of Galactic rotation, both with respect to the Sun. are responsible for the Hercules stream, while unstable orbits have been shown to be responsible for the observed gap between the main mode and Hercules. This implies a pattern speed of the order of $\Omega_{\mathrm{b}} \approx 1.9 \Omega_{0}$. With such a fast bar model, it was subsequently shown that the Oort constants could be reproduced (Minchev et al. 2007), and that the expected azimuthal velocity location of Hercules as a function of Galactocentric radius complied with data from the RAVE survey (Antoja et al. 2014). The double-peak structure identified by Famaey et al. (2008) within Hercules is however not reproduced by a bar-only model, and has been suspected to be linked to another perturbation, most probably spiral arms. Finally, another feature which might presumably be associated with the bar is located at $(U, V) \simeq(75,-55) \mathrm{km} \mathrm{s}^{-1}$ (Dehnen 1998), denoted hereafter as the 'horn' of the velocity distribution.

Note that the precise location of Hercules in velocity space after correction for the reflex Solar motion is slightly dependent on the Solar motion itself. Considerable debate still exists regarding this motion, especially in the $V$ direction. By extrapolating the asymmetric drift relation to zero velocity dispersion, Dehnen \& Binney (1998) estimated that the Sun moves in the direction of Galactic rotation only slightly faster than the circular velocity, namely $V_{\odot}=$ $5.25 \mathrm{~km} \mathrm{~s}^{-1}$, while they estimated $U_{\odot}=10 \mathrm{~km} \mathrm{~s}^{-1}$ by simply assuming no mean radial motion from the Local Standard of rest itself. More recent discrepant values for the solar motion include Schönrich (2012) estimating $U_{\odot}=14 \mathrm{~km} \mathrm{~s}^{-1}$ and $V_{\odot}=12 \mathrm{~km} \mathrm{~s}^{-1}$, as well as Bovy et al. (2015) estimating $U_{\odot}=10 \mathrm{~km} \mathrm{~s}^{-1}$ and $V_{\odot}=24 \mathrm{~km} \mathrm{~s}^{-1}$. Hereafter, we will display the position of the two peaks (H1 and H2) of the Hercules stream in peculiar velocity space $(u, v)$ for these different values of the solar motion. The local circular speed $v_{0}$ and Sun's distance from the centre of the Galaxy $R_{0}$ will be fixed such that $v_{0}+V_{\odot}=30.24 \mathrm{~km} \mathrm{~s}^{-1} \mathrm{kpc}^{-1} \times R_{0}$ (Reid \& Brunthaler 2004). In the Schönrich (2012) and Bovy et al. (2015) cases we use the values for $v_{0}$ (238 and $218 \mathrm{~km} \mathrm{~s}^{-1}$, respectively), and $R_{0}(8.27 \mathrm{kpc}$ and $8 \mathrm{kpc}$, respectively) proposed by the authors. In the Dehnen \& Binney (1998) case we assume $R_{0}=8 \mathrm{kpc}$, which corresponds to $v_{0}=236.67 \mathrm{~km} \mathrm{~s}^{-1}$. Contrary to most previous studies, we will concentrate on the form of the phase-space DF expected in the Solar neighbourhood from perturbation theory, in the spirit of M16, rather than on individual orbits.

\section{PERTURBED DISTRIBUTION FUNCTIONS}

\subsection{General case: first order response}

Let $(R, \phi, z)$ be a cylindrical coordinate system with origin at the centre of the Milky Way. The bulk of the mass of the Galaxy is associated with an axisymmetric gravitational potential $\Phi_{0}(R, z)$, and it is well-known that realistic galactic potentials are close to integrable ones. In this case, the natural canonical coordinates for dynamics are the actionangle variables (see, e.g., Binney \& Tremaine 2008; Fouvry et al. 2015), (J, $\boldsymbol{\theta})$, where $\mathbf{J}$ are integrals of motion in $\Phi_{0}$. Thanks to the Jeans theorem, a stellar population described by an axisymmetric phase-space distribution function (DF) $f_{0}=f_{0}(\mathbf{J})$ is in equilibrium.

Here, we follow the approach of M16 (see also 
Kalnajs 1971, Carlberg \& Sellwood 1985) in which a nonaxisymmetric perturbing potential $\Phi_{1}(R, \phi, z, t)$ is expanded in a Fourier series of the angles $\boldsymbol{\theta}$ as

$$
\Phi_{1}(\mathbf{J}, \boldsymbol{\theta}, t)=\operatorname{Re}\left\{g(t) h(t) \sum_{\mathbf{n}} c_{\mathbf{n}}(\mathbf{J}) \mathrm{e}^{\mathrm{in} \cdot \boldsymbol{\theta}}\right\},
$$

where $g(t)$ controls the growth of the perturbation with time, and $h(t)$ is a periodic sinusoidal function, of frequency $\omega_{\mathrm{p}}$, which accounts for the perturbing potential rotating at a fixed pattern speed. Typically, $\omega_{\mathrm{p}}=-m \Omega_{\mathrm{p}}$ where $m$ is the multiplicity (or azimuthal wavenumber) of the perturbing potential and $\Omega_{\mathrm{p}}$ its pattern speed, and $h(t)=\exp \left(\mathrm{i} \omega_{\mathrm{p}} t\right)$.

We assume that $g(t)$ is a well behaved function, that the perturbation and its time derivatives were null far back in time $\left(g^{(k)}(-\infty)=0\right)$, and that the perturbation has constant amplitude at the present time $\left(g^{(0)}(t)=1\right.$, and $g^{(k)}(t)=0$ for $\left.k=1, \ldots, \infty\right)$. Using the linearized Boltzmann equation, the linear response to first order of the stellar equilibrium distribution $f_{0}$ to the perturbing potential $\Phi_{1}$ has been shown by M16 to be $f=f_{0}+f_{1}$ with

$$
f_{1}(\mathbf{J}, \boldsymbol{\theta}, t)=\operatorname{Re}\left\{\frac{\partial f_{0}}{\partial \mathbf{J}}(\mathbf{J}) \cdot \sum_{\mathbf{n}} \mathbf{n} c_{\mathbf{n}}(\mathbf{J}) \frac{h(t) \mathrm{e}^{\mathrm{i} \mathbf{n} \cdot \boldsymbol{\theta}}}{\mathbf{n} \cdot \boldsymbol{\omega}+\omega_{\mathrm{p}}}\right\} .
$$

The predictions of this perturbed DF have been directly compared to test-particle simulations in M16, and showed remarkable agreement in terms of the moments of the DF. One might however wonder what is gained by our analytical treatment of the problem compared to the results of these test-particle simulations. The big difference is that, contrary to such simulations, our analytical DFs will in the future allow us to fit the data directly, with a few fitting parameters in the perturbing potential as well as in the axisymmetric DF, by performing a maximum-likelihood estimate of these parameters based on actual kinematical data for a large set of individual stars.

The DFs computed from perturbation theory however diverge for resonant orbits, for which $\mathbf{n} \cdot \boldsymbol{\omega}(\mathbf{J})+\omega_{\mathrm{p}}=0$. Resonances are responsible for the 'trapping' of orbits, which renders the former linear treatment inappropriate near to resonances. We will identify the resonantly trapped orbits in Section 3.3.3 in order to display their location in local velocity space. But, first, we will expand our perturbative treatment to second order, to make sure that we are not missing any peculiar behavior of the DF with our first order treatment of the problem.

\subsection{General case: second order response}

In the previous section we derived the linear response $f_{1}$ of the DF to the perturbing potential $\Phi_{1}$. 'Linear' means that if $\left|\Phi_{1} / \Phi_{0}\right| \sim \varepsilon \ll 1$, then $f_{1} \in \mathcal{O}(\varepsilon)$, and we neglect all higher order terms. We can expand the DF to the second order as $f=f_{0}+f_{1}+f_{2}+\mathcal{O}\left(\varepsilon^{3}\right)$, where $f_{2} \in \mathcal{O}\left(\varepsilon^{2}\right)$. Plugging this expression in the collisionless Boltzmann equation, and grouping together the $\mathcal{O}(\varepsilon), \mathcal{O}\left(\varepsilon^{2}\right)$, and $\mathcal{O}\left(\varepsilon^{3}\right)$ terms, we obtain the linear equation for $f_{1}$ that was used to obtain Eq. (2), i.e.,

$$
\frac{\mathrm{d} f_{1}}{\mathrm{~d} t}+\left[f_{0}, \Phi_{1}\right]=0
$$

and the second order equation

$$
\frac{\mathrm{d} f_{2}}{\mathrm{~d} t}+\left[f_{1}, \Phi_{1}\right]=0 .
$$

Therefore,

$$
f_{2}(\mathbf{J}, \boldsymbol{\theta}, t)=-\int_{-\infty}^{t} \mathrm{~d} t\left[f_{1}, \Phi_{1}\right] .
$$

We can rewrite $f_{2}$ as $f_{2}=\tilde{f}_{2}-\hat{f}_{2}$, where

$$
\tilde{f}_{2}(\mathbf{J}, \boldsymbol{\theta}, t) \equiv \int_{-\infty}^{t} \mathrm{~d} t \frac{\partial f_{1}}{\partial \mathbf{J}} \cdot \frac{\partial \Phi_{1}}{\partial \boldsymbol{\theta}},
$$

and

$$
\hat{f}_{2}(\mathbf{J}, \boldsymbol{\theta}, t) \equiv \int_{-\infty}^{t} \mathrm{~d} t \frac{\partial \Phi_{1}}{\partial \mathbf{J}} \cdot \frac{\partial f_{1}}{\partial \boldsymbol{\theta}}
$$

\subsection{Rotating bar case}

\subsubsection{First order}

As Weinberg (1994) and Dehnen (2000), we assume that the perturbing potential due to the Galactic bar behaves, outside from the bar itself, as a quadrupole. Furthermore, we are only interested here in the response inside the $z=0$ Galactic plane, i.e. we write for the bar potential

$$
\Phi_{1}(R, \phi, t)=\operatorname{Re}\left\{\Phi_{\mathrm{a}}(R) \mathrm{e}^{\mathrm{i} m\left(\phi-\phi_{\mathrm{b}}-\Omega_{\mathrm{b}} t\right)}\right\},
$$

where $m=2, \Omega_{\mathrm{b}}$ is the pattern speed of the perturber in the bar case, $\phi_{\mathrm{b}}$ is the angle between the Sun and the long axis of the bar, and

$$
\Phi_{\mathrm{a}}(R)=-\alpha_{\mathrm{b}} \frac{v_{0}^{2}}{3}\left(\frac{R_{0}}{R_{\mathrm{b}}}\right)^{3} \times \begin{cases}\left(R / R_{\mathrm{b}}\right)^{-3} & R \geqslant R_{\mathrm{b}}, \\ 2-\left(R / R_{\mathrm{b}}\right)^{3} & R<R_{\mathrm{b}}\end{cases}
$$

where $(R, \phi)$ are the Galactocentric radius and azimuth, $R_{\mathrm{b}}$ is the length of the bar, and $\alpha_{\mathrm{b}}$ represents the maximum ratio between the bar and axisymmetric background radial forces at the Sun's position $R=R_{0}$ (see also Monari et al. 2015. 2016b).

Using Eq. 22, and making use of the epicyclic approximation, M16 derived the form of $f_{1}$ for any rotating Fourier mode of the kind Eq. (8), for stellar populations close to the Galactic plane and on low eccentricity orbits. For stars orbiting on the Galactic plane (i.e. with $z=0$ and $v_{z}=0$ ), $f_{1}$ reads

$$
f_{1}=\operatorname{Re}\left\{\sum_{j=-1}^{1} c_{j m} \mathrm{~F}_{j m} \mathrm{e}^{\mathrm{i}\left[j \theta_{R}+m\left(\theta_{\phi}-\phi_{\mathrm{b}}-\Omega_{\mathrm{b}} t\right)\right]}\right\},
$$

with

$$
\begin{aligned}
c_{j m}\left(J_{R}, J_{\phi}\right) \equiv & {\left[\delta_{j 0}+\delta_{|j| 1} \frac{m}{2} \operatorname{sgn}(j) \gamma e\right] \Phi_{\mathrm{a}}\left(R_{\mathrm{g}}, 0\right) } \\
& -\delta_{|j| 1} \frac{R_{\mathrm{g}}}{2} e \frac{\partial \Phi_{\mathrm{a}}}{\partial R}\left(R_{\mathrm{g}}, 0\right)
\end{aligned}
$$

and

$$
\mathrm{F}_{j m}\left(J_{R}, J_{\phi}\right) \equiv \frac{j \frac{\partial f_{0}}{\partial J_{R}}+m \frac{\partial f_{0}}{\partial J_{\phi}}}{j \kappa+m\left(\omega_{\phi}-\Omega_{\mathrm{b}}\right)} .
$$

Hereabove, we denote the circular and epicyclic frequencies with the usual notation $\Omega$ and $\kappa$, which are evaluated 
at the guiding radius $R_{\mathrm{g}}\left(J_{\phi}\right)$, defined as the radius where $R_{\mathrm{g}}^{2} \Omega\left(R_{\mathrm{g}}\right)=J_{\phi}$. We also define $\gamma \equiv 2 \Omega / \kappa$, the eccentricity $e\left(J_{R}, J_{\phi}\right) \equiv \sqrt{2 J_{R} /\left(\kappa R_{\mathrm{g}}^{2}\right)}$, and the azimuthal frequency $\omega_{\phi}\left(J_{R}, J_{\phi}\right) \equiv \Omega+\left(\mathrm{d} \kappa / \mathrm{d} J_{\phi}\right) J_{R}$.

Within the epicyclic approximation, we can relate the actions $\left(J_{R}, J_{\phi}, \theta_{R}, \theta_{\phi}\right)$ to the usual cylindrical phase-space coordinates $\left(R, \phi, v_{R}, v_{\phi}\right)$ through

$$
\begin{array}{lll}
J_{R}=\frac{v_{R}^{2}}{2 \kappa}+\frac{\kappa\left(R-R_{\mathrm{g}}\right)}{2}, & J_{\phi}=R v_{\phi}, \\
\theta_{R}=\tan ^{-1}\left(-\frac{v_{R}}{\kappa\left(R-R_{\mathrm{g}}\right)}\right), & \theta_{\phi}=\phi+\Delta \phi,
\end{array}
$$

where

$$
\Delta \phi \equiv-\frac{\gamma}{R_{\mathrm{g}}} \sqrt{\frac{2 J_{R}}{\kappa}} \sin \theta_{R}-\frac{J_{R}}{2} \frac{\mathrm{d} \ln \kappa}{\mathrm{d} J_{\phi}} \sin \left(2 \theta_{R}\right) .
$$

Using the above relations, the phase-space DF $f=f_{0}+$ $f_{1}$ can be expressed as a function of the usual phase-space coordinates, i.e. $f=f\left(R, \phi, v_{R}, v_{\phi}\right)$.

\subsubsection{Second order}

We now compute the second order response $f_{2}$ from $f_{1}$ and $\Phi_{1}$ in the case case of a quadrupole potential like the one of Eq. (8). Once the derivatives in Eqs. (6)-(7) are calculated, one needs to solve simple integrals of sinusoidal functions, in the same fashion as in the $f_{1}$ case. Then, after defining the two-dimensional vectors $\mathbf{n} \equiv(j, m)$, and $\mathbf{N} \equiv\left(j^{\prime}, m\right)$, Eqs. 67- 7] become,

$$
\begin{gathered}
\tilde{f}_{2}(\mathbf{J}, \boldsymbol{\theta})=-\frac{1}{2} \sum_{j, j^{\prime}=-1}^{1} \frac{\partial}{\partial \mathbf{J}}\left(c_{j m} F_{j m}\right) \cdot \mathbf{N} c_{j^{\prime} m} \operatorname{Im}\left\{\mathcal{S}_{j j^{\prime}}^{-}\right\} \\
\hat{f}_{2}(\mathbf{J}, \boldsymbol{\theta})=-\frac{1}{2} \sum_{j, j^{\prime}=-1}^{1} \mathbf{n} c_{j m} F_{j m} \cdot \frac{\partial c_{j^{\prime} m}}{\partial \mathbf{J}} \operatorname{Im}\left\{\mathcal{S}_{j j^{\prime}}^{+}\right\}
\end{gathered}
$$

where

$$
\begin{array}{r}
\mathcal{S}_{j j^{\prime}}^{ \pm}(\mathbf{J}, \boldsymbol{\theta}) \equiv-\mathrm{i}\left[\frac{\mathrm{e}^{\mathrm{i}\left[(\mathbf{n}+\mathbf{N}) \cdot \boldsymbol{\theta}-2 m \phi_{\mathrm{b}}-2 m \Omega_{\mathrm{b}} t\right]}}{(\mathbf{n}+\mathbf{N}) \cdot \boldsymbol{\omega}-2 m \Omega_{\mathrm{b}}}\right. \\
\left. \pm\left(1-\delta_{j, j^{\prime}}\right) \frac{\mathrm{e}^{\mathrm{i}\left(j-j^{\prime}\right) \theta_{R}}}{\left(j-j^{\prime}\right) \kappa}\right]
\end{array}
$$

With these expressions, we can now calculate the response to the bar up to second-order $f=f_{0}+f_{1}+f_{2}$, which we will plot in velocity space at a given point in the Galactic plane.

Note that higher-order terms with $m>2$ would in principle also appear in the expansion of the bar potential of Eq. (8) for more complex bar shapes than a pure quadrupole. These additional terms would have their own associated perturbed DF and are not considered here. These DFs would in principle be of second or higher order compared to the quadrupole amplitude. As we will show in the next section, the second order effects computed hereabove for the quadrupole case are subdominant indeed compared to the first order response, and this should thus be the case for these additional terms in the perturbing potential too.

\subsubsection{Resonantly trapped orbits}

It can immediately be seen from Eq. 12 that the linear response $f_{1}$ to the rotating perturbation $\Phi_{1}$ diverges for resonant orbits whose frequencies $\kappa$ and $\omega_{\phi}$ are such that

$$
l \kappa+m\left(\omega_{\phi}-\Omega_{\mathrm{b}}\right)=0,
$$

with $l=0$ (corotation resonance), or $l= \pm 1$ (Lindblad resonances). These are the first-order resonances.

Insight on the dynamics of a star near a resonance can be obtained using a canonical transformation of coordinates defined by the type- 2 generating function (e.g., Weinberg 1994)

$$
S=\left[l \theta_{R}+m\left(\theta_{\phi}-\Omega_{\mathrm{b}} t\right)\right] J_{\mathrm{s}}+\theta_{R} J_{\mathrm{f}}
$$

The new angles and actions $\left(\theta_{\mathrm{f}}, \theta_{\mathrm{s}}, J_{\mathrm{f}}, J_{\mathrm{s}}\right)$ are related to the old ones by

$$
\begin{array}{ll}
\theta_{\mathrm{s}}=l \theta_{R}+m\left(\theta_{\phi}-\Omega_{\mathrm{b}} t\right), & J_{\phi}=m J_{\mathrm{s}}, \\
\theta_{\mathrm{f}}=\theta_{R}, & J_{R}=l J_{\mathrm{s}}+J_{\mathrm{f}} .
\end{array}
$$

In these new canonical coordinates, the motion is described by the Hamiltonian (e.g. Binney \& Tremaine 2008, Appendix D.4.6)

$$
H^{\prime}\left(\theta_{\mathrm{f}}, \theta_{\mathrm{s}}, J_{\mathrm{f}}, J_{\mathrm{s}}\right)=H\left(\theta_{R}, \theta_{\phi}, J_{R}, J_{\phi}, t\right)+\frac{\partial S}{\partial t},
$$

Since $\partial S / \partial t=-m \Omega_{\mathrm{b}} J_{\mathrm{s}}$, and rewriting $H$ as a function of the new coordinates $\left(\theta_{\mathrm{f}}, \theta_{\mathrm{s}}, J_{\mathrm{f}}, J_{\mathrm{s}}\right), H^{\prime}$ reads

$$
H^{\prime}=H_{0}+\operatorname{Re}\left\{\sum_{j=-1}^{1} c_{j m} \mathrm{e}^{\mathrm{i}\left[(j-l) \theta_{\mathrm{f}}+\theta_{\mathrm{s}}\right]}\right\}-m \Omega_{\mathrm{b}} J_{\mathrm{s}},
$$

where $H_{0}=H_{0}\left(J_{R}, J_{\phi}\right)$ is the Hamiltonian of the unperturbed axisymmetric system and the $c_{j m}\left(J_{R}, J_{\phi}\right)$ coefficients are the Fourier coefficients from Eq. [11). In this case, $J_{R}$ and $J_{\phi}$ have to be understood as functions of $\left(J_{\mathrm{f}}, J_{\mathrm{s}}\right)$, as given by the canonical transformation in Eq. 20.2

The angle $\theta_{\mathrm{s}}$ is usually called 'slow angle' because near a resonance, for an orbit in the axisymmetric background potential, it evolves very slowly by definition of the resonance in Eq. (18), while $\theta_{\mathrm{f}}$ is called the 'fast angle'. Since $\theta_{\mathrm{f}}$ evolves much faster than $\theta_{\mathrm{s}}$, we can average $H^{\prime}$ along $\theta_{\mathrm{f}}$ (the averaging principle, e.g., Arnold 1978, Weinberg 1994 . Binney \& Tremaine 2008), to obtain

$$
\overline{H^{\prime}}=H_{0}\left(J_{\mathrm{f}}, J_{\mathrm{s}}\right)-m \Omega_{\mathrm{b}} J_{\mathrm{s}}+\operatorname{Re}\left\{c_{l m}\left(J_{\mathrm{f}}, J_{\mathrm{s}}\right) \mathrm{e}^{\mathrm{i} \theta_{\mathrm{s}}}\right\} .
$$

Since $\dot{J}_{\mathrm{f}}=-\partial \overline{H^{\prime}} / \partial \theta_{\mathrm{f}}=0, J_{\mathrm{f}}$ is an integral of motion, and the motion can be described only in the $\left(\theta_{\mathrm{s}}, J_{\mathrm{s}}\right)$ plane. We can further write the Hamilton equations as,

$$
\begin{aligned}
& \dot{\theta}_{\mathrm{s}}=\frac{\partial \overline{H^{\prime}}}{\partial J_{\mathrm{s}}}=\Omega_{\mathrm{s}}+\operatorname{Re}\left\{\frac{\partial c_{l m}}{\partial J_{\mathrm{s}}} \mathrm{e}^{\mathrm{i} \theta_{\mathrm{s}}}\right\}, \\
& \dot{J}_{\mathrm{s}}=-\frac{\partial \overline{H^{\prime}}}{\partial \theta_{\mathrm{s}}}=-\operatorname{Re}\left\{\mathrm{i} c_{l m} \mathrm{e}^{\mathrm{i} \theta_{\mathrm{s}}}\right\},
\end{aligned}
$$

where $\Omega_{\mathrm{s}}$ is the angular frequency associated to $\theta_{\mathrm{s}}$ in the unperturbed axisymmetric Hamiltonian in the rotating frame

\footnotetext{
2 Notice that for any given orbit $H^{\prime}$ takes a value that we can call $E_{\mathrm{J}}$, which is an integral of motion of the perturbed system. $E_{\mathrm{J}}$ is the energy in the frame of reference rotating with the perturbation, and is usually known as the 'Jacobi integral'.
} 
$H_{0}-\Omega_{b} J_{\phi}$. Dropping all the terms which are $\mathcal{O}\left(\varepsilon^{2}\right)$, we get:

$$
\ddot{\theta_{\mathrm{s}}} \approx \operatorname{Re}\left\{\mathrm{i}\left(\Omega_{\mathrm{s}} \frac{\partial c_{l m}}{\partial J_{\mathrm{s}}}-\frac{\partial \Omega_{\mathrm{s}}}{\partial J_{\mathrm{s}}} c_{l m}\right) \mathrm{e}^{\mathrm{i} \theta_{\mathrm{s}}}\right\} .
$$

Note that if we Taylor expand the function $\Omega_{\mathrm{s}}\left(\partial c_{l m} / \partial J_{\mathrm{s}}\right)-$ $\left(\partial \Omega_{\mathrm{s}} / \partial J_{\mathrm{s}}\right) c_{l m}$ about $J_{\mathrm{s}}$ and again drop all the terms that are $\mathcal{O}\left(\varepsilon^{2}\right)$, Eq. 25 becomes a one-dimensional pendulum equation which can be rewritten as $\ddot{\theta}_{\mathrm{s}}=-\mathrm{d} V_{\mathrm{p}}\left(\theta_{\mathrm{s}}\right) / \mathrm{d} \theta_{\mathrm{s}}$, where

$$
V_{\mathrm{p}}\left(\theta_{\mathrm{s}}\right)=\operatorname{Re}\left\{\left(\frac{\partial \Omega_{\mathrm{s}}}{\partial J_{\mathrm{s}}} c_{l m}-\Omega_{\mathrm{s}} \frac{\partial c_{l m}}{\partial J_{\mathrm{s}}}\right) \mathrm{e}^{\mathrm{i} \theta_{\mathrm{s}}}\right\}
$$

is the pendulum potential and

$$
E_{\mathrm{p}}={\dot{\theta_{\mathrm{s}}}}^{2} / 2+V_{\mathrm{p}}\left(\theta_{\mathrm{s}}\right)
$$

its energy. The maximum of the potential $V_{\max }$ is

$$
V_{\max }=\left|\frac{\partial \Omega_{\mathrm{s}}}{\partial J_{\mathrm{s}}} c_{l m}-\Omega_{\mathrm{s}} \frac{\partial c_{l m}}{\partial J_{\mathrm{s}}}\right| .
$$

The angle $\theta_{\mathrm{s}}$ describes the precession angle of the orbit with respect to the closed resonant orbit in the frame of reference rotating with the perturbation, while $\theta_{\mathrm{f}}$ is the motion of the star along its orbit itself (Weinberg 1994, Binney \& Tremaine 2008). For $E_{\mathrm{p}}<V_{\max }$ the angle $\theta_{\mathrm{s}}$ librates back and forward between two values, around the closed orbit, while for $E_{\mathrm{p}}>V_{\max }, \theta_{\mathrm{s}}$ circulates. Orbits that have $E_{\mathrm{p}}<V_{\max }$ are the orbits 'trapped at the resonance', while the circulating orbits with $E_{\mathrm{p}} \gg V_{\max }$ can be fully described by the perturbative treatment explained in the previous sections.

In the following we will display the zone of local velocity space corresponding to orbits trapped by the first-order resonances. This trapping will affect the actual density of stars in velocity space in the trapped zone compared to our models, but will not strongly affect the general shape of velocity space itself.

\section{SLOW BAR MODELS}

We will now explore the actual response of stars in the solar neighbourhood to a bar perturbation.

Let $R_{0}$ be the Sun's Galactocentric radius and $\Omega_{0}=$ $\Omega\left(R_{0}\right)$ the local rotation frequency. As outlined in Section 1 recent models of the distribution and kinematics of stars the inner part of the Milky Way (Long et al. 2013 Portail et al. 2015, Wegg et al. 2015) as well as models of the gas kinematics (Sormani et al. 2015. Li et al. 2016) favour pattern speeds estimates of the bar much lower $\left(\Omega_{0} \lesssim \Omega_{\mathrm{b}} \lesssim 1.45 \Omega_{0}\right)$ than the ones that for more than a decade were considered as settled $\left(1.8 \Omega_{0} \lesssim \Omega_{\mathrm{b}} \lesssim 2 \Omega_{0}\right)$. While the old estimates predict the OLR to be in proximity of the Solar neighbourhood, the new estimates rather predict that stars in the Sun's vicinity would be influenced by the bar's corotation (CR), or at least that the Sun is located well inside the OLR. In this Section we analyze what this would mean in terms of the shape of the DF in the Solar neighbourhood.

Using a common notation in Galactic Astronomy, for each point of the Galactic plane $(R, \phi)$, we define the peculiar velocity coordinates ${ }^{3}$ of stars, $u \equiv-v_{R}$ and $v \equiv$ $v_{\phi}-v_{\mathrm{c}}(R)$, where the circular velocity $v_{\mathrm{c}}(R) \equiv R \Omega(R)$. As

3 Note that in general, when we consider volumes of finite non- in M16, we choose a quasi-isothermal DF for $f_{0}(\mathbf{J})$, first introduced by Binney \& McMillan (2011) as a modification of the DF defined by Binney $(2010)$, and reducing to a Schwarzschild DF in the epicyclic approximation (see M16 for the details of the DF parameters).

Hereafter, when our perturbed $f_{1}$ (and $f_{2}$ ) diverges due to our treatment to only 1st order (or 2nd order), we simply choose to set $f_{1}=\operatorname{sgn}\left(f_{1}\right)\left|f_{0}\right|\left(f_{2}=\operatorname{sgn}\left(f_{2}\right)\left|f_{0}\right|\right)$ whenever $f_{0}<\left|f_{1}\right|\left(f_{0}<\left|f_{2}\right|\right)$ due to $\left|f_{1}\right|$ (or $\left.\left|f_{2}\right|\right)$ being larger than $f_{0}$ near first-order or second-order resonances. A detailed treatment of the behaviour of the perturbed DF in the trapped regions will be the topic of further work, but we already clearly identify the regions affected by first-order resonant trapping thanks to the formalism developed in Section 3.3 .3 The regions of trapping at the resonances, where $E_{\mathrm{p}}<V_{\max }$ (see Section 3.3.3), will be surrounded on the figures by red contours in the CR case, and by blue contours in the OLR case.

\subsection{First order response in bar-only models}

In Fig. 1 we plot the value of the distribution function $f=$ $f_{0}+f_{1}$ as a function of the velocities $(u, v)$ expressed as a function of the local circular speed $v_{0}$, for stars at $(R, \phi)=$ $\left(R_{0}, 0\right)$ and $v_{z}=0$, i.e. orbiting on the Galactic plane and passing in the Solar neighbourhood at the present time. We consider a range of pattern speeds coherent with the recent estimates by Portail et al. (2015), Sormani et al. (2015) and Li et al. (2016), namely $\Omega_{\mathrm{b}}=\left[\Omega_{0}, 1.45 \Omega_{0}\right]$.

To obtain this figure, we used the parameters $\phi_{\mathrm{b}}=25^{\circ}$ (which controls the angle between the bar's long axis and the line connecting the Sun and the Galactic centre), and $\alpha_{\mathrm{b}}=0.01$. We set $R_{\mathrm{b}}=0.625 R_{0}$, but also tried a case where the bulk of the mass is in the inner parts of the bar, $R_{\mathrm{b}}=$ $0.44 R_{0}$, and found qualitatively exactly the same patterns in velocity space.

As can be seen from the trapped regions in Fig. 11 for $\Omega_{\mathrm{b}}<1.27 \Omega_{0}$, the DF is not influenced by the OLR. The $\mathrm{CR}$ has instead an influence for $v<0$ : the velocity distribution is clearly split into two parts that we call the 'low- $v$ ' and 'high $-v$ ' modes. Stars in the high- $v$ mode have $u \lesssim 0$ (i.e. they have the slight tendency to move outwards in the Galaxy), while stars in the low $-v$ mode have $u \gtrsim 0$. The observed $\mathrm{H} 1$ and $\mathrm{H} 2$ velocity peaks of the Hercules moving group, corrected for the Sun's motion according to different estimates (see Section 2), are represented by the red points for the estimate of Dehnen \& Binney (1998), by the blue points for the estimate of Schönrich (2012), and by the green points for the Bovy et al. (2015) estimate. As is evident from this figure, even if the low $-v$ mode is formed because of the CR, for none of the Sun's motion estimates is its position compatible with the actual position of Hercules. Actually, the stars of the Hercules moving group have on average $u<0$, while the low- $v$ mode generated by the CR has

zero size, $u \neq-v_{R}$ and $v \neq v_{\phi}-v_{\mathrm{c}}(R)$. In fact, $(u, v)$ in Galactic Astronomy refer to the Cartesian velocities in the Local Standard of Rest (Binney \& Tremaine 2008). However, in the case of velocity space at a given point in configuration space, like we consider in this work, $u=-v_{R}$ and $v=v_{\phi}-v_{\mathrm{c}}(R)$ is a good approximation. 

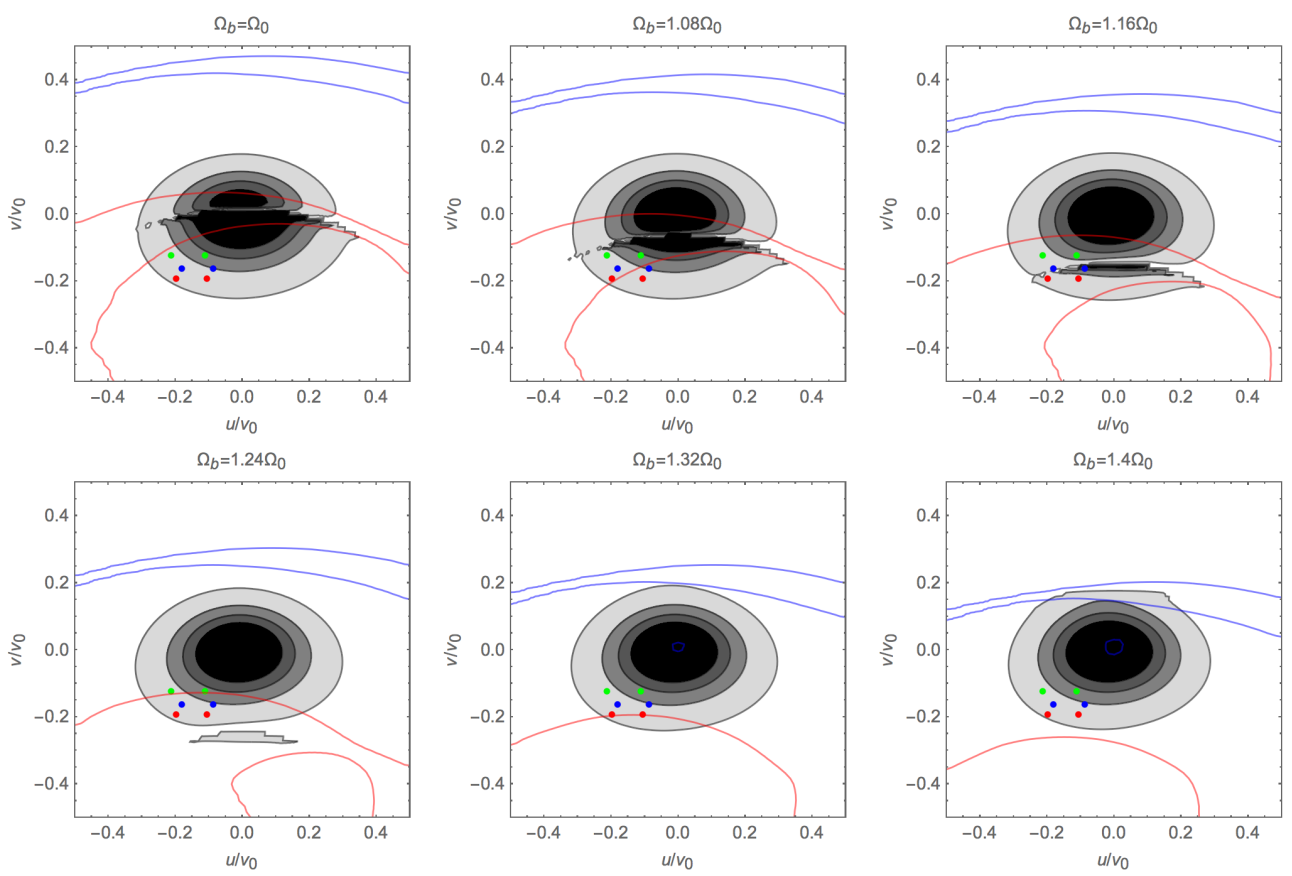

Figure 1. The local stellar velocity distribution perturbed to the linear order (i.e. the phase-space DF $f=f_{0}+f_{1}$, with $f_{0}$ the axisymmetric DF of M16 - namely the quasi-isothermal DF first introduced by Binney \& McMillan (2011) as a modification of the DF defined by Binney (2010)- and $f_{1}$ given by Eq. 110$)$ in the $(u, v)$ plane at $(R, \phi, z)=\left(R_{0}, 0,0\right)$ for different bar models with low pattern speed $\left(\Omega_{0} \leqslant \Omega_{\mathrm{b}} \leqslant 1.4 \Omega_{0}\right)$. In this and all the figures of this work, the contours include $34,50,68$, and 90 per cent of the stars respectively. Here the local maximum ratio at $R_{0}$ between the bar and axisymmetric background radial forces is $\alpha_{\mathrm{b}}=0.01$. The colored points represent the observed Hercules moving group peaks H1 and H2 as estimated by Famaey et al. (2008), corrected for the Sun's motion in the estimates of Dehnen \& Binney (1998) (red points), Schönrich (2012) (blue points), and Bovy et al. (2015) (green points). The red (blue) contours delimit the region of resonant trapping by the CR (OLR), i.e. orbits with $E_{\mathrm{p}}<V_{\max }$ as defined in Eq. 27) and Eq. 28). At $\Omega_{\mathrm{b}} \simeq 1.45 \Omega_{0}$ (Sormani et al. 2015), the zone of influence of the CR (red contours) is clearly moving away from the bulk of stars in velocity space, whilst the OLR (blue contours) only has an influence at high $v$, far from the actual location of the Hercules moving group in local velocity space (coloured points). For $\Omega_{\mathrm{b}} \simeq 1.2 \Omega_{0}$ (Li et al. 2016), the CR of the bar does create a bimodality at the right location in $v$, but not in $u$.
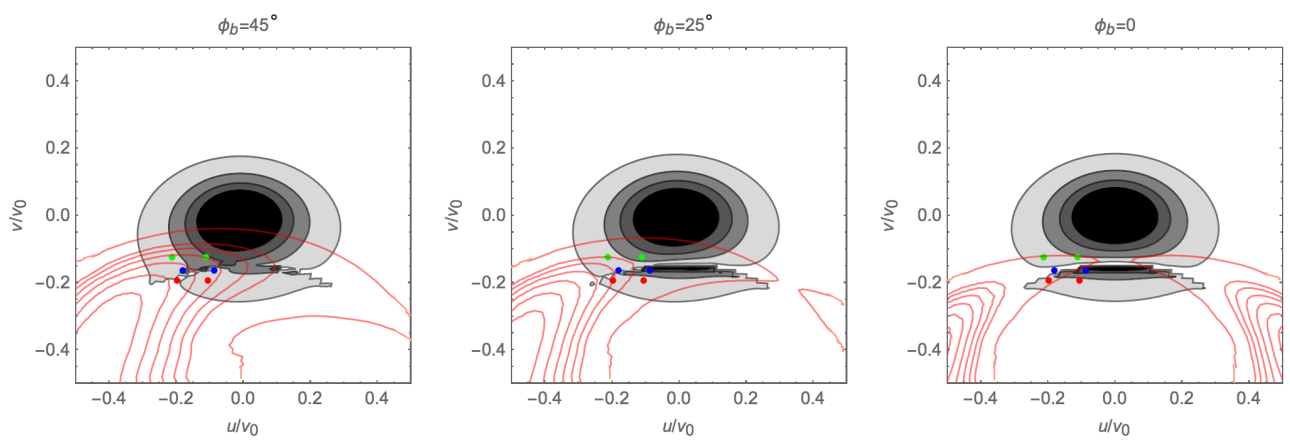

Figure 2. As in Fig. 1. but this time fixing the pattern speed to $\Omega_{\mathrm{b}}=1.16 \Omega_{0}$, and varying the angle $\phi_{\mathrm{b}}$. This does not help, as to get the bimodality at the right location to reproduce Hercules (colored points), one would need a negative $\phi_{\mathrm{b}}$ clearly excluded from the photometric structure of the Milky Way bar. The inner red contours represent different values of $\eta=E_{\mathrm{p}} / V_{\max }$, for orbits trapped at the bar's CR. In particular, they correspond to $\eta_{i}=\eta_{\min }+i \Delta \eta$, for $i=1, \ldots, 6, \eta_{\min }$ the minimum of $\eta$, and $\Delta \eta=(1-\eta) / 10$. The trapping onto closed orbits (smallest $\eta$ ) is far from the bulk of stars in velocity space.

$u \gtrsim 0$. On the other hand, when the pattern speed increases to $\Omega_{\mathrm{b}} \geqslant 1.27 \Omega_{0}$ the OLR has a slight influence only to a few stars with very high- $v$, not at all in the relevant region of velocity space for the range of pattern speed considered in this section.

Observations clearly show that $\phi_{\mathrm{b}} \geqslant 0$ (Binney et al. 1997). However, a definitive estimate of the bar's angle is still missing. In Fig. 2 we thus explore the effect of varying the bar angle in the range $\phi_{\mathrm{b}}=\left[0,45^{\circ}\right]$. While, because of the symmetry of the model, for $\phi_{\mathrm{b}}=0$ the DF is exactly symmetric with respect to $u=0$, increasing $\phi_{\mathrm{b}}$ moves the low $-v$ mode to larger $u$, thus increasing its distance from the actual position of the Hercules moving group, and making the situation worse. 


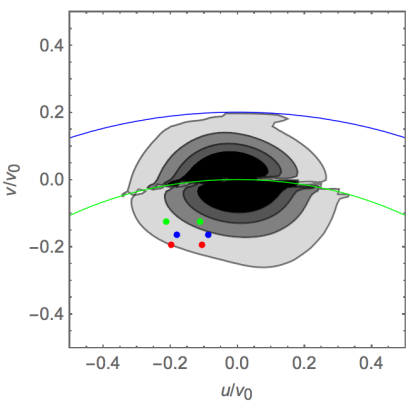

Figure 3. Illustration of the effect of the second-order response to the bar potential in an extreme unrealistic case with $\alpha_{\mathrm{b}}=0.1$. The figure displays the velocity distribution in the $(u, v)$ plane at $(R, \phi, z)=\left(R_{0}, 0,0\right)$ including only the second order DF response (i.e. $f=f_{0}+f_{2}$ ) for $\Omega_{\mathrm{b}}=1.36 \Omega_{0}$. The blue line displays the location of the OLR, and the green line the outer ultraharmonic resonance (OUHR). Here one can see that not only is the effect negligible for a realistic bar strength (i.e., in reality, one would have $\alpha_{\mathrm{b}} \ll 1$ ), the location of the effect in velocity space also does not correspond to the Hercules moving group.

An alternative possibility would nevertheless be that the region of strongest trapping, by construction not well taken into account by our linear model, would correspond to the location of Hercules. We thus also plot on Fig. 2 the isocontours of the energy of the pendulum $E_{\mathrm{p}}$ (see Section 3.3.3): we can see that the region of strongest trapping close to the resonant closed orbit is outside of the bulk of stars in velocity space, and would correspond to stars orbiting in the plane but with extremely high eccentricity.

No feature in our modelled local velocity space can thus account for the Hercules stream to first order in bar-only models.

\subsection{Second order response in bar-only models}

Fig. 3 shows $f$ when $\Omega_{\mathrm{b}}$ is chosen so that $R_{0}$ is at the radius of the outer ultraharmonic resonance $R_{\mathrm{OUH}}$, defined as the radius $R$ where $\kappa\left(R_{\mathrm{OUH}}\right)+4\left[\Omega\left(R_{\mathrm{OUH}}\right)-\Omega_{\mathrm{b}}\right]=0$. Near to $R_{\mathrm{OUH}}$ we have the strongest effect of the outer ultraharmonic resonance,

$$
\kappa+4\left(\omega_{\phi}-\Omega_{\mathrm{b}}\right)=0
$$

The second order expansion of the collisionless Boltzmann equation (Section 3.3.2 takes into account the ultraharmonic resonances, as appears clearly in the denominators of Eqs. 15-16. For amplitudes of the bar potential similar to those that we used in the previous Sections, the effects of the second order resonance are small, and not enough to appreciate the effects of the outer ultraharmonic resonance on the distribution function $f$. Therefore, in Fig. 3 we plot an extreme case, where the amplitude of the bar radial force is as large as 10 percent of that of the axisymmetric background $\left(\alpha_{\mathrm{b}}=0.1\right)$, and we plot only the effects of $f_{2}$. This extreme case is fully unrealistic for the Milky Way, but clearly shows that the effect of the ultraharmonic resonance of the bar cannot explain the formation of the Hercules moving group: while $f$ is split in two parts in $v$, the low- $v$ mode of the distribution has in average positive $u$, again contrary to the observed behaviour of the Hercules moving group.

\subsection{Models with spiral arms}

We now analyze the combined effects of the bar potential and a spiral pattern potential. Away from resonance overlap regions, the combined linear response $f_{1}$ is then simply the sum of the response due to the bar and that of the spiral arms (calculated in the same way as for the bar, but for a different $\left.\Phi_{\mathrm{a}}\right)$.

A question is whether spiral arms could be responsible for the Hercules moving group. In their work, Li et al. (2016) propose a best-fit model of the kinematics of the Milky Way, with a perturbing potential composed by a slow bar $\left(\Omega_{\mathrm{b}}=\right.$ $33 \mathrm{~km} \mathrm{~s}^{-1} \mathrm{kpc}^{-1}$ ), and two $m=2$ spiral arms patterns, displaced by $\Delta \phi_{\mathrm{sp}}=20.25^{\circ}$ in azimuth, rotating with an angular frequency $\Omega_{\mathrm{sp}}=23 \mathrm{~km} \mathrm{~s}^{-1} \mathrm{kpc}^{-1}$.

We reproduce a spiral potential similar to that of $\mathrm{Li}$ et al. (2016) by considering two Fourier modes with $m=2$, as in Eq. (8). In this case $\Omega_{\mathrm{p}}=\Omega_{\mathrm{sp}}=0.84 \Omega_{0}$, and

$$
\Phi_{\mathrm{a}}(R)=\alpha_{\mathrm{sp}} \Phi_{0}\left(R_{0}, 0\right) \mathrm{e}^{-\mathrm{i} m \ln \left(R / R_{\mathrm{sp}}\right) / \tan p},
$$

where $R_{\mathrm{s}}=0.125 R_{0}$. As a reference value we assume $\alpha_{\mathrm{sp}}=$ 0.005 (Siebert et al. 2012). The locus of the arms and the pitch angle $p$ are also like in Li et al. (2016). The pattern speed of the bar is set at $\Omega_{\mathrm{b}}=1.2 \Omega_{0}$, and $R_{\mathrm{b}}=0.625 R_{0}$. We show the results of this model to the linear order in Fig. 4. for different values of the spiral arm amplitude. It is clear from this figure that, up to the linear order response, this model does not describe in a satisfying way the kinematics of the Solar neighbourhood.

In Fig. 5 we consider the second order response, both to the spiral arms and bar perturbation. The calculation of $f_{2}$ in the case of the spiral arms is more cumbersome than in the bar case, since the Fourier coefficients $c_{j m}$ are in this case complex numbers, quadrupling the number of terms of $f_{2}$. The $\tilde{f}_{2}$ and $\hat{f}_{2}$ components of Eqs. (6)- (7) become

$$
\begin{aligned}
\tilde{f}_{2}(\mathbf{J}, \boldsymbol{\theta})= & -\sum_{j, j^{\prime}=-1}^{1} \frac{\mathbf{N}}{2} \cdot[ \\
& +\frac{\partial}{\partial \mathbf{J}} \operatorname{Re}\left\{c_{j m} F_{j m}\right\} \operatorname{Re}\left\{c_{j^{\prime} m}\right\} \operatorname{Im}\left\{\mathcal{S}_{j j^{\prime}}^{-}\right\} \\
& +\frac{\partial}{\partial \mathbf{J}} \operatorname{Re}\left\{c_{j m} F_{j m}\right\} \operatorname{Im}\left\{c_{j^{\prime} m}\right\} \operatorname{Re}\left\{\mathcal{S}_{j j^{\prime}}^{+}\right\} \\
& +\frac{\partial}{\partial \mathbf{J}} \operatorname{Im}\left\{c_{j m} F_{j m}\right\} \operatorname{Re}\left\{c_{j^{\prime} m}\right\} \operatorname{Re}\left\{\mathcal{S}_{j j^{\prime}}^{-}\right\} \\
& \left.-\frac{\partial}{\partial \mathbf{J}} \operatorname{Im}\left\{c_{j m} F_{j m}\right\} \operatorname{Im}\left\{c_{j^{\prime} m}\right\} \operatorname{Im}\left\{\mathcal{S}_{j j^{\prime}}^{+}\right\}\right] \\
\hat{f}_{2}(\mathbf{J}, \boldsymbol{\theta})= & -\sum_{j, j^{\prime}=-1} \frac{\mathbf{n}}{2} \cdot[ \\
& +\operatorname{Re}\left\{c_{j m} F_{j m}\right\} \frac{\partial}{\partial \mathbf{J}} \operatorname{Re}\left\{c_{j^{\prime} m}\right\} \operatorname{Im}\left\{\mathcal{S}_{j j^{\prime}}^{+}\right\} \\
& +\operatorname{Im}\left\{c_{j m} F_{j m}\right\} \frac{\partial}{\partial \mathbf{J}} \operatorname{Re}\left\{c_{j^{\prime} m}\right\} \operatorname{Re}\left\{\mathcal{S}_{j j^{\prime}}^{-}\right\} \\
& +\operatorname{Re}\left\{c_{j m} F_{j m}\right\} \frac{\partial}{\partial \mathbf{J}} \operatorname{Im}\left\{c_{j^{\prime} m}\right\} \operatorname{Re}\left\{\mathcal{S}_{j j^{\prime}}^{+}\right\} \\
& \left.-\operatorname{Im}\left\{c_{j m} F_{j m}\right\} \frac{\partial}{\partial \mathbf{J}} \operatorname{Im}\left\{c_{j^{\prime} m}\right\} \operatorname{Im}\left\{\mathcal{S}_{j j^{\prime}}^{-}\right\}\right] .
\end{aligned}
$$

The result is qualitatively fully unchanged, the second order response of both the bar and spirals being very small in the region of interest. One can see that the spiral indeed distorts the velocity distribution somewhat in the right direction, 

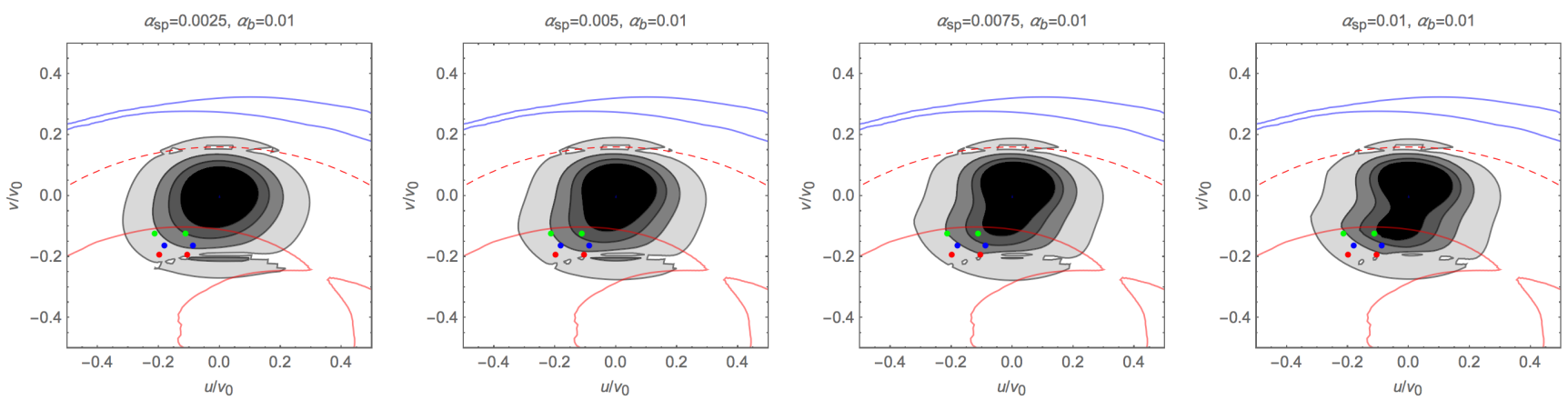

Figure 4. Linear response of the DF (i.e. $\left.f=f_{0}+f_{1}\right)$ in the $(u, v)$ plane at $(R, \phi, z)=\left(R_{0}, 0,0\right)$, for a model with both a slow bar and a spiral pattern similar to the one of Li et al. (2016). It includes two $m=2$ spiral arms modes rotating with pattern speed $\Omega_{\mathrm{sp}}=0.84 \Omega_{0}$, and a bar with $\Omega_{\mathrm{b}}=1.2 \Omega_{0}$ and $\phi_{\mathrm{b}}=27^{\circ}$. Different panels display the response for different amplitudes of the spiral arms. The red (blue) solid contours delimit the regions of resonant trapping to the bar's CR (OLR). The red dashed line represents the CR of the spiral arms.
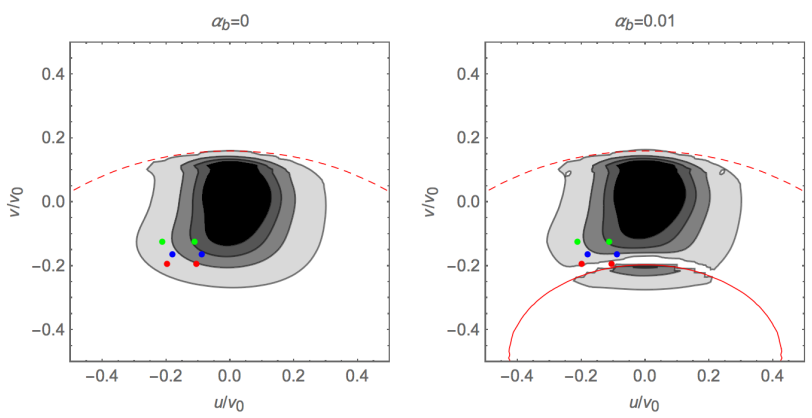

Figure 5. Effects of the second order response $\left(f=f_{0}+f_{1}+\right.$ $f_{2}$ ) on the model shown in Fig. 4 for $\alpha_{\mathrm{sp}}=0.005$, without (left panel) and with (right panel) the bar. The red solid (dashed) line represents the $\mathrm{CR}$ of the bar (spiral arms).

but the effect is very limited and does not at all render the velocity space bimodal as observed. This slight distortion of velocity space is more likely related to the observed Hyades and Ursa Major moving groups than to Hercules.

\section{FAST BAR MODELS}

In the models of this Section we show how our models reproduce the traditional results expected in the Solar neighbourhood for a fast bar. We use $\Omega_{\mathrm{b}}=1.89 \Omega_{0}$, following the estimates of Antoja et al. (2014). This pattern speed corresponds to $R_{\mathrm{OLR}}=0.9 R_{0}$, where $R_{\mathrm{OLR}}$ is defined as $R$ satisfying

$$
2\left[\Omega\left(R_{\mathrm{OLR}}\right)-\Omega_{\mathrm{b}}\right]+\kappa\left(R_{\mathrm{OLR}}\right)=0 .
$$

In Fig. 6 we show the results for $\phi_{\mathrm{b}}=25^{\circ}$ and different values of the amplitude of the perturbation in the range $\alpha=[0.005,0.02]$. The results keep in the account both the linear and the quadratic response of the disc DF, $f_{1}$ and $f_{2}$ respectively. In this case, $f$ is split in two parts as observed in Section 2, Stars in the low- $v$ (high- $v$ ) mode have $\omega_{\phi}<\Omega_{\mathrm{b}}-\kappa / 2\left(\omega_{\phi}>\Omega_{\mathrm{b}}-\kappa / 2\right)$, and tend to move outwards (inwards) in the Galaxy, i.e. they have $u<0(u>0)$. This is a direct consequence of the linear effects of the bar on the stars' orbits. In particular, the orbits of stars with guiding centre inside (outside) the OLR become elongated perpendicularly (along) the bar (Binney \& Tremaine 2008). Fig. 6 in agreement with the results of several of the numerical simulations of the effects of the bar in the Solar neighbourhood, starting from the pioneering work of Dehnen (2000). The stars forming the low- $v$ velocity mode are usually associated with the Hercules moving group, and the gap between this moving group and the main velocity mode in the Solar neighbourhood were used by Antoja et al. (2014) to estimate the pattern speed of the bar, assuming that the gap is due to the bar's OLR.

Increasing the bar's strength moves the stars closer to the resonance curve. Moreover, stars in the low- $v$ (high- $v$ ) mode travel faster outwards (inwards) in the Galaxy. Interestingly, we see that while all the estimates are compatible with the identification of the low $v$ mode with the Hercules moving group, the estimates with higher Sun's tangential velocity $V_{\odot}$ (Bovy et al. 2015) is globally favoured by the fast bar models considered here, and also favour models with a stronger bar.

In Fig. 7 we show the variation of the DF as a function of $\phi_{\mathrm{b}}$, keeping constant $R$. Also in this case the second order effects are taken into account. We present only positive values of $\phi_{\mathrm{b}}$ as observations show that this is the case in the Milky Way (Binney et al. 1997). Moreover, because of the symmetry of the DF in Eq. (10), the case of negative angles is readily obtained simply flipping the signs of the $u$ velocities in Fig. 7. At $\phi=0$ the DF is completely symmetric with the respect of $u=0$, while increasing the angle $\phi_{\mathrm{b}}$ increases the number of stars with negative (positive) $u$ for the low (high) $v$ velocity mode.

Then, using the form of the spiral arms potential in Eq. 30, we study the combined effects of the fast bar and spiral arms up to the second order.

In Fig. 8 we show $f$ at $(R, \phi)=\left(R_{0}, 0\right)$ in the plane for the combination of the fast bar and two spiral arms models. The figures keep in account the first and second order effects, both for the bar and the spiral arms. As bar parameters we use $\Omega_{\mathrm{b}}=1.89 \Omega_{0}, \phi_{\mathrm{b}}=25^{\circ}$, and $\alpha_{\mathrm{b}}=0.01$. The first spiral arm model is formed by the two $m=2$ modes with the same locus of the spiral arms as Li et al. (2016). The second model is only one $m=2$ mode, representing the old stellar arms, with the parameters taken from Siebert et al. (2012). The 

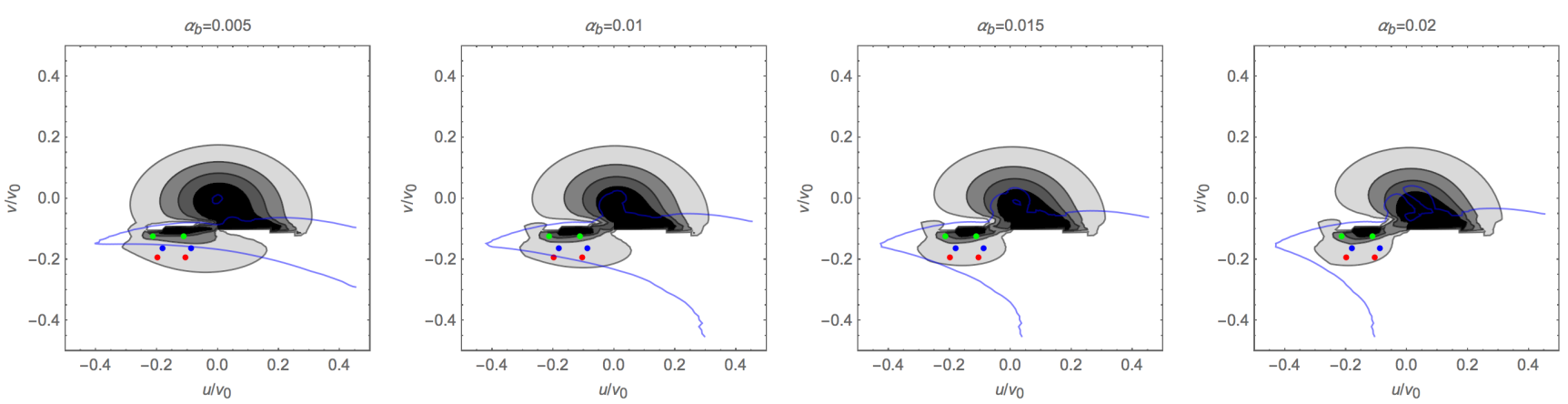

Figure 6. Velocity distribution to the second order (i.e. $\left.f=f_{0}+f_{1}+f_{2}\right)$ in the $(u, v)$ plane at $(R, \phi, z)=\left(R_{0}, 0,0\right)$ for a fast bar model $\Omega_{\mathrm{b}}=1.89 \Omega_{0}$ and $\phi_{\mathrm{b}}=25^{\circ}$. We consider different bar's amplitudes $\alpha_{\mathrm{b}}$. The blue contours represent regions of trapping to the bar's OLR.
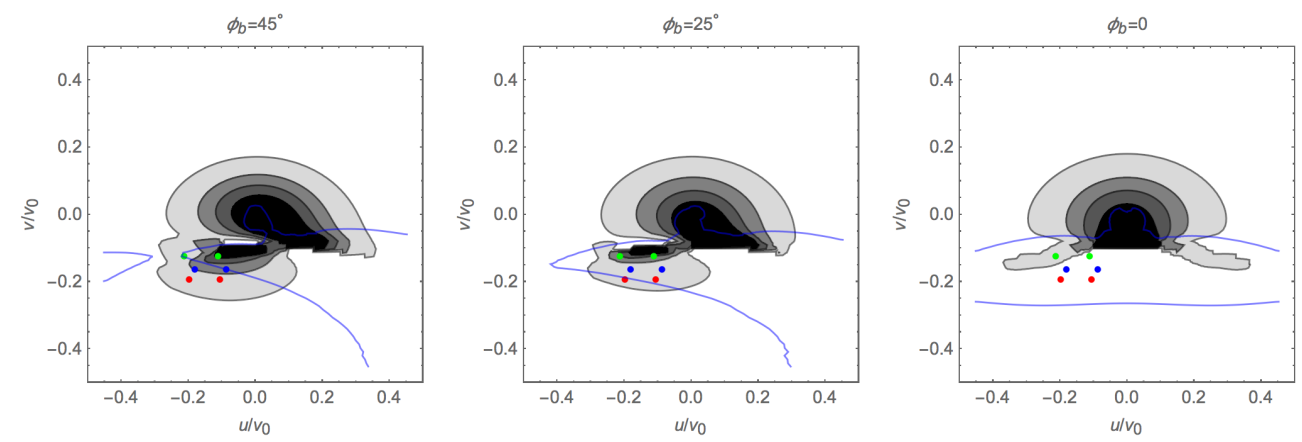

Figure 7. As in Fig. 6, but keeping fixed the bar's amplitude to $\alpha_{\mathrm{b}}=0.01$, and varying the angle $\phi_{\mathrm{b}}$.
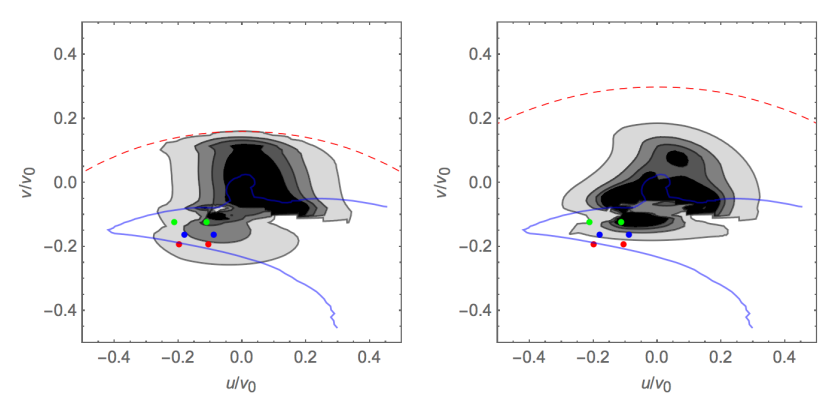

Figure 8. Second order response of the DF (i.e. $f=f_{0}+f_{1}+f_{2}$ ) for the spiral arms models of Li et al. (2016) (left panel) and Siebert et al. (2012) (right panel), and a fast bar with $\Omega_{\mathrm{b}}=$ $1.89 \Omega_{0}$ and $\phi_{\mathrm{b}}=25^{\circ}$ in the $(u, v)$ plane at $(R, \phi, z)=\left(R_{0}, 0,0\right)$.

pattern speed is in the former case $\Omega_{\mathrm{sp}}=0.84 \Omega_{0}(\mathrm{Li}$ et al. 2016), and in the latter $\Omega_{\mathrm{sp}}=0.69 \Omega_{0}$ (Siebert et al. 2012).

The comparison with Fig. 8 shows that the Siebert et al. (2012) spiral arms increase the probability to find stars in the low- $v$ mode, and deforms the shape of both the low and high- $v$ mode, and in particular the latter. The deformation in the high- $v$ mode slightly resembles the Hyades moving group overdensity in the Solar neighbourhood. In the same region of the velocity space we can notice the effect of the inner ultraharmonic resonance, that creates a small gap in the high- $v$ mode. Several authors suggested (e.g., Quillen \& Minchev 2005, Pompéia et al. 2011) that the inner ultra- harmonic resonance of the spiral arms could be the cause of the Hyades moving group. Our treatment seems to suggest that this resonance would be too weak to influence alone the velocity distribution in such a significant way. However, given its vicinity with the bar OLR, it could be that the resonance overlap would render our treatment not appropriate, and the coupling effects important (e.g., Monari et al. 2016b). A fully proper treatment near resonances, including resonance overlaps, will be the topic of further work. We however note that the simulations of Monari et al. (2016b) did not show significant differences for the in-plane motions between the coupled simulation and the linear combination of simulations with a single perturber. The difference was much more pronounced in terms of vertical motions, which we do not consider here.

Then, in Fig. 9 we reproduce the central plot $\left(\phi_{\mathrm{b}}=25^{\circ}\right)$ of Fig. 7. and we superpose on top of it the contours of pendulum energy $E_{\mathrm{p}}$ for the OLR (blue contours). We see that the contours representing the strongest trapping coincide with the 'horn' region that we mentioned in Section 2 Indeed, it seems that the overdensity that several authors found in their simulations with the same fast bar models (e.g., Dehnen 2000) could be explained by the orbits trapped to the OLR resonance. Monari (2014) came to the same conclusion, using however a different method, the numerical Fourier analysis of orbits in the simulations.

Finally, we note that we assumed throughout this paper a rather simple form for the background axisymmetric DF $f_{0}$. We thus also show the results (Fig. 10 for two different values of the pattern speed for a more involved axisymmetric 


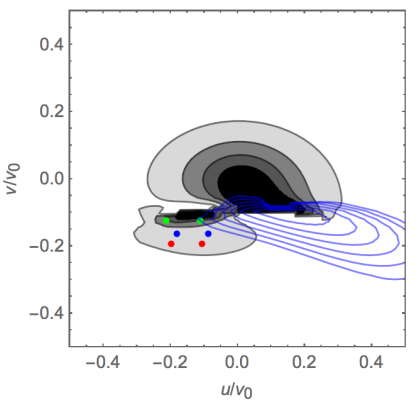

Figure 9. As in the central panel of Fig. 7, but this time including the normalized energy of the pendulum $\eta=E_{\mathrm{p}} / V_{\max }$, for orbits trapped at the bar's OLR. In particular, the blue contours correspond to $\eta_{i}=\eta_{\min }+i \Delta \eta$, for $i=1, \ldots, 6, \eta_{\min }$ the minimum of $\eta$, and $\Delta \eta=(1-\eta) / 10$.
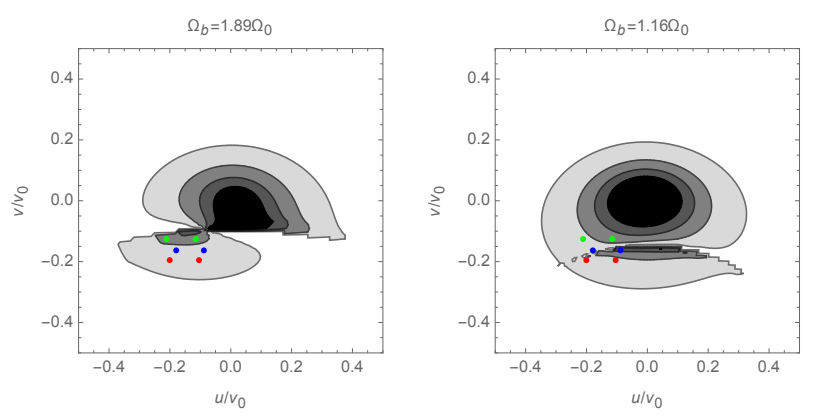

Figure 10. Velocity distribution to the first order (i.e. $f=f_{0}+$ $\left.f_{1}\right)$ in the $(u, v)$ plane at $(R, \phi, z)=\left(R_{0}, 0,0\right)$ for a fast $\left(\Omega_{\mathrm{b}}=\right.$ $\left.1.89 \Omega_{0}\right)$ and slow bar model $\left(\Omega_{\mathrm{b}}=1.16 \Omega_{0}\right), \phi_{\mathrm{b}}=25^{\circ}$, and $\alpha_{\mathrm{b}}=$ 0.01 , where $f_{0}$ and $f_{1}$ are given in Eqs. 34- 37 .

DF, i.e.,

$$
f_{0}=\sum_{i} w_{i} f_{0, i}
$$

Here, $f_{0, i}$ are quasi-isothermal DFs of the same kind of those used in M16 and in the rest of this work, but each of them has a different velocity dispersion $\tilde{\sigma}_{R, i}\left(R_{0}\right)$ (see equation 42 of M16). Each $f_{0, i}$ represents a stellar population with a different age $\tau_{i}$, which is related to the velocity dispersion via

$$
\tilde{\sigma}_{R}\left(R_{0}\right)=\sigma_{0}\left(\frac{\tau_{i}+\tau_{1}}{\tau_{\mathrm{m}}+\tau_{1}}\right)^{\beta},
$$

where $\sigma_{0}=40.1 \mathrm{~km} \mathrm{~s}^{-1}, \tau_{1}=0.01 \mathrm{Gyr}, \tau_{\mathrm{m}}=10 \mathrm{Gyr}$, and $\beta=0.33$ (Binney 2012). This means that a stellar population born now has a dispersion $\tilde{\sigma}_{R}\left(R_{0}\right) \approx 4 \mathrm{~km} \mathrm{~s}^{-1}$, whilst a 1 Gyr old population has a dispersion $\tilde{\sigma}_{R}\left(R_{0}\right) \approx$ $19 \mathrm{~km} \mathrm{~s}^{-1}$, and a 10 Gyr old population a dispersion $\tilde{\sigma}_{R}\left(R_{0}\right)=40.1 \mathrm{~km} \mathrm{~s}^{-1}$. The weights $w_{i}$ are also inspired by equation 12 of Binney (2012), and are related to $\tau_{i}$ by

$$
w_{i}=\frac{\Delta \tau_{i} \mathrm{e}^{\gamma \tau_{i}}}{\sum_{i} \Delta \tau_{i} \mathrm{e}^{\gamma \tau_{i}}}
$$

where $\Delta \tau_{i}$ are age intervals in the range $\left[0, \tau_{\mathrm{m}}\right]$ for thin disc stars taken from Table 3 of Robin et al. (2003); $\tau_{i}$ is the central age value of each bin, and $\gamma=0.117$ (see Aumer \& Binney 2009).

The total linear response $f_{1}$ to the bar perturbation is simply the weighted sum of the linear responses of each sub-population $f_{1, i}$, i.e.,

$$
f_{1}=\sum_{i} w_{i} f_{1, i} .
$$

Fig. 10 shows clearly that the results of our analysis in the rest of this work, which uses a single population of velocity dispersion $\tilde{\sigma}_{R}\left(R_{0}\right)=35 \mathrm{~km} \mathrm{~s}^{-1}$ to study the effects of the bar's pattern speed, still hold in the case of a more complex DF, representing a reasonable superposition of stellar populations of different ages and velocity dispersions.

\section{CONCLUSIONS}

We presented the first application of the formalism developed in M16 to calculate, through perturbation theory, the effects of a non-axisymmetric gravitational disturbance on an initially axisymmetric DF, $f_{0}(\mathbf{J})$, describing the phasespace density of stars in a collisionless stellar system (i.e., governed by the collisionless Boltzmann equation).

We extended the M16 formalism to second order (Section 3), and concentrated on the effects of the Galactic bar on the DF in the Solar neighbourhood. We checked whether a slow bar with pattern speed $\Omega_{0} \lesssim \Omega_{\mathrm{b}} \lesssim 1.45 \Omega_{0}$ could reproduce the observed bimodality of local velocity space. We concluded that no feature in our modelled local velocity space could account for the observed bimodality (Section 4). We checked whether second order effects, or the additional effects of spiral arms, could help, and did not find any configuration reproducing the bimodality. A fast bar with $\Omega_{\mathrm{b}} \approx 1.9 \Omega_{0}$, on the other hand, explains it nicely (Section 5). Bland-Hawthorn \& Gerhard (2016) fixed their final value of the bar's pattern speed at $\Omega_{\mathrm{b}}=(1.48 \pm 0.31) \Omega_{0}$. Here we estimated that, if Hercules is created by the bar's OLR, the pattern speed of the bar cannot be less than $\Omega_{\mathrm{b}} \approx 1.8 \Omega_{0}$ to be compatible with the measured density peaks of the Hercules moving group.

In Section 3.3.3 and in all our figures, we also identified the regions of resonant trapping in phase-space. This trapping should affect the actual density of stars in the trapped zone compared to the analytical models presented here, but does not strongly affect the general distortion of phase-space itself, as numerical particle test simulations with adiabatic growth of the bar (e.g., Monari et al.2014) give the same result as our fast bar models for the shape of the bimodality in local velocity space. We note that, while such forward testparticle simulations can serve as benchmarks to test analytical models like those presented here (see M16), they do not allow to directly fit the data. Actually, the main motivation of models based on analytical DFs is that they will indeed allow to fit the data directly, with a few fitting parameters in the perturbing potential as well as in the axisymmetric DF, by performing a maximum-likelihood estimate of these parameters based on actual kinematical data for a large set of individual stars. However, in order to perform such a fully quantitative fit, our method will have to be extended to better treat the DF for resonantly trapped orbits. This will be the topic of a forthcoming paper.

Concerning the bimodality, let us also note that we did not try every possible spiral arm configuration here, and cannot yet strictly exclude that a similar structure as the 
locally observed bimodality could be the result of spirals. Our results are generally in line with the N-body simulations of Quillen et al. (2011) in which velocity distributions created from regions just outside the bar's OLR more closely resembled that seen in the solar neighbourhood. Nevertheless, close inspection of the velocity distributions at other radii in these simulations reveal spiral-related features which also slightly resemble the Hercules stream, albeit at angles to the bar which do not correspond to the present orientation of the bar in the Milky Way. Also, Grand et al. (2014) showed that the outward radial migrators behind their corotating spiral arms display lower- $v$ and negative- $u$ velocity (see their Fig. 4), hence providing a possible explanation which will have to be inspected closely in the future. In any case, the future DR2 and DR3 data releases from Gaia (Gaia Collaboration 2016) should allow a detailed investigation of phase-space structure outside of the Solar neighbourhood, at different Galactic radii and azimuths, and test our present conclusions about the pattern speed of the bar, since any possible spiral-related features in velocity space would not follow the same evolution at different radii and azimuths. Such a test might actually already be possible by combining the Gaia DR1 with existing spectroscopic surveys. We also note that the metallicity patterns in local stellar velocity space seem to also support our fast bar models (Antoja et al. 2016, in preparation).

The three-dimensional density of red clump giants in the inner Galaxy nevertheless clearly indicate the existence of a long, flat structure, oriented at an angle of $\phi_{\mathrm{b}} \sim 27^{\circ}$ from the Galactic centre-Sun direction and reaching out to a radius $\sim 5 \mathrm{kpc}$. The most natural explanation would be that this structure is not a long bar but rather a loosely wound spiral coupled to the end of the bar. If it has a pattern speed only somewhat smaller than the central bar, it could be a good candidate to explain the observed doublepeak aspect of the Hercules stream, which is not reproduced even in our fast bar models. On the other hand, it is known that small nuclear bars with faster pattern speed than the main bar can be long-lived in numerical simulations including a gaseous component, even without resonance overlaps or mode coupling, if star formation remains moderately active in the region of the nuclear bar (e.g., Wozniak 2015). However, we are not aware of any simulation reproducing a stable long bar with lower pattern speed than its central counterpart and similar in size to the structure observed in the inner $5 \mathrm{kpc}$ of the Milky Way (hence about twice the disc scale-length). We would thus a priori favour a loosely wound spiral structure to explain the photometric observations.

\section{ACKNOWLEDGEMENTS}

We thank the anonymous referee for the constructive reports. This work has been supported by a postdoctoral grant from the Centre National d'Etudes Spatiales (CNES) for GM.

\section{REFERENCES}

Antoja T. et al., 2014, A\&A, 563, A60
Arnold V. I., 1978, Mathematical methods of classical mechanics, Arnold, V. I., ed.

Athanassoula E., 2005, MNRAS, 358, 1477

Athanassoula E., Bienayme O., Martinet L., Pfenniger D., 1983, A\&A, 127, 349

Aumer M., Binney J. J., 2009, MNRAS, 397, 1286

Bensby T., Oey M. S., Feltzing S., Gustafsson B., 2007, ApJ, 655, L89

Binney J., 2010, MNRAS, 401, 2318

Binney J., 2012, MNRAS, 426, 1324

Binney J., Gerhard O., Spergel D., 1997, MNRAS, 288, 365

Binney J., Gerhard O. E., Stark A. A., Bally J., Uchida K. I., 1991, MNRAS, 252, 210

Binney J., McMillan P., 2011, MNRAS, 413, 1889

Binney J., Tremaine S., 2008, Galactic Dynamics: Second Edition, Binney, J. \& Tremaine, S., ed. Princeton University Press

Bissantz N., Englmaier P., Gerhard O., 2003, MNRAS, 340, 949

Blaauw A., 1970, in IAU Symposium, Vol. 38, The Spiral Structure of our Galaxy, Becker W., Kontopoulos G. I., eds., p. 199

Bland-Hawthorn J., Gerhard O., 2016, arXiv:1602.07702

Bovy J., 2010, ApJ, 725, 1676

Bovy J., Bird J. C., García Pérez A. E., Majewski S. R., Nidever D. L., Zasowski G., 2015, ApJ, 800, 83

Carlberg R. G., Sellwood J. A., 1985, ApJ, 292, 79

Combes F., Debbasch F., Friedli D., Pfenniger D., 1990, A\&A, 233, 82

de Vaucouleurs G., 1964, in IAU Symposium, Vol. 20, The

Galaxy and the Magellanic Clouds, Kerr F. J., ed., p. 195

Dehnen W., 1998, AJ, 115, 2384

Dehnen W., 1999a, AJ, 118, 1190

Dehnen W., 1999b, ApJ, 524, L35

Dehnen W., 2000, AJ, 119, 800

Dehnen W., Binney J. J., 1998, MNRAS, 298, 387

Eggen O. J., 1958, MNRAS, 118, 154

Famaey B., Jorissen A., Luri X., Mayor M., Udry S., Dejonghe H., Turon C., 2005, A\&A, 430, 165

Famaey B., Pont F., Luri X., Udry S., Mayor M., Jorissen A., 2007, A\&A, 461, 957

Famaey B., Siebert A., Jorissen A., 2008, A\&A, 483, 453

Fouvry J.-B., Pichon C., Prunet S., 2015, MNRAS, 449, 1967

Gaia Collaboration, 2016, arXiv:1609.04153

Grand R. J. J., Kawata D., Cropper M., 2014, MNRAS, 439, 623

Kalnajs A. J., 1971, ApJ, 166, 275

Kapteyn J. C., 1905, Reports of the British Association for the Advancement of Science, Section A, 257-65, 264

Li Z., Gerhard O., Shen J., Portail M., Wegg C., 2016, ApJ, 824, 13

Long R. J., Mao S., Shen J., Wang Y., 2013, MNRAS, 428, 3478

Minchev I., Nordhaus J., Quillen A. C., 2007, ApJ, 664, L31

Monari G., 2014, PhD thesis, Rijksuniversiteit Groningen Monari G., Famaey B., Siebert A., 2015, MNRAS, 452, 747 Monari G., Famaey B., Siebert A., 2016a, MNRAS, 457, 2569

Monari G., Famaey B., Siebert A., Grand R. J. J., Kawata D., Boily C., 2016b, MNRAS, 461, 3835 
Monari G., Helmi A., Antoja T., Steinmetz M., 2014, A\&A, 569, A69

Mühlbauer G., Dehnen W., 2003, A\&A, 401, 975

Pompéia L. et al., 2011, MNRAS, 415, 1138

Portail M., Wegg C., Gerhard O., Martinez-Valpuesta I., 2015, MNRAS, 448, 713

Proctor R. A., 1869, Proceedings of the Royal Society of London Series I, 18, 169

Quillen A. C., Dougherty J., Bagley M. B., Minchev I., Comparetta J., 2011, MNRAS, 417, 762

Quillen A. C., Minchev I., 2005, AJ, 130, 576

Ramya P., Reddy B. E., Lambert D. L., Musthafa M. M., 2016, MNRAS, 460, 1356

Reid M. J., Brunthaler A., 2004, ApJ, 616, 872

Robin A. C., Reylé C., Derrière S., Picaud S., 2003, A\&A, 409, 523

Schönrich R., 2012, MNRAS, 427, 274

Siebert A. et al., 2012, MNRAS, 425, 2335

Sormani M. C., Binney J., Magorrian J., 2015, MNRAS, 454, 1818

Soubiran C., Girard P., 2005, A\&A, 438, 139

Wegg C., Gerhard O., 2013, MNRAS, 435, 1874

Wegg C., Gerhard O., Portail M., 2015, MNRAS, 450, 4050

Weinberg M. D., 1994, ApJ, 420, 597

Wozniak H., 2015, A\&A, 575, A7 